\title{
Boomerang and Slide-Rotational Analysis of the SM3 Hash Function
}

\author{
Aleksandar Kircanski ${ }^{1}$, Yanzhao Shen ${ }^{2}$, Gaoli Wang ${ }^{2,3, \star}$, and Amr M. Youssef ${ }^{1}$ \\ 1 Concordia University \\ Concordia Institute for Information Systems Engineering \\ Montreal, Quebec, Canada \\ 2 Donghua University \\ School of Computer Science and Technology, Shanghai, China \\ 3 State Key Laboratory of Information Security \\ Institute of Software, Chinese Academy of Sciences, Beijing, China \\ wanggaoli@dhu.edu.cn
}

\begin{abstract}
SM3 is a hash function, designed by Xiaoyun Wang et al. and published by the Chinese Commercial Cryptography Administration Office for the use of electronic authentication service system. The design of SM3 builds upon the design of the SHA-2 hash function, but introduces additional strengthening features. In this paper, we present boomerang distinguishers for the SM3 compression function reduced to 32 steps out of 64 steps with complexity $2^{14.4}, 33$ steps with complexity $2^{32.4}, 34$ steps with complexity $2^{53.1}$ and 35 steps with complexity $2^{117.1}$. Examples of zero-sum quartets for the 32-step and 33-step SM3 compression function are provided. We also point out a slide-rotational property of SM3-XOR, which exists due to the fact that constants used in the steps are not independent.
\end{abstract}

Keywords: Cryptanalysis, Boomerang attack, Rotational attack, Slide attack, SM3.

\section{Introduction}

In December of 2007, the Chinese National Cryptographic Administration Bureau released the specification of a Trusted Cryptography Module detailing a cryptoprocessor to be used within the Trusted Computing framework in China. The module specifies a set of cryptographic algorithms that includes the SMS4 block cipher, the SM2 asymmetric algorithm and SM3, a new cryptographic hash function designed by Xiaoyun Wang et al. [1]. The design of SM3 resembles the design of SHA-2 but includes additional fortifying features such as feeding two message-derived words into each step, as opposed to only one in the case of SHA-2.

The only previous work that we are aware of on the analysis of SM3 has been presented by Zou et al. [2] at ICISC 2011 where a preimage attack on step-reduced SM3 is provided. In particular, Zou et al. presented attacks on SM3 reduced to 30 steps, starting from the 7-th step, with time complexity $2^{249}$ and 28 steps, starting from the 1 -st step with time complexity $2^{241.5}$.

\footnotetext{
^ Corresponding author.
} 
The use of zero-sums as distinguishers have been introduced by Aumasson et al. [3]. The boomerang attack [4], originally introduced for block ciphers, has been adapted to the hash function setting independently by Biryukov et al. [5], and Lamberger and Mendel [6]. In particular, in [5], a distinguisher for the 7-round BLAKE-32 was provided, whereas in [6] a distinguisher for the 46-step SHA-2 compression function was provided. The latter SHA-2 result was extended to 47 steps in [7]. In [8], Mendel and Nad presented boomerang distinguishers for the SIMD-512 compression function. Sasaki [9] gave a boomerang distinguisher on the full compression function of 5-pass HAVAL. Sasaki also proposed a 2-dimension sums attack on 48-step RIPEMD-128 and 42-step RIPEMD-160 in [10]. Boomerang distinguishers have also been applied to Skein and Threefish. In [11] Aumasson et al. proposed a related-key boomerang distinguisher on 34-step Skein and a known-related-key boomerang distinguisher on 35-step Skein. In [12], Leurent and Roy showed that, under some conditions, three independent paths instead of two can be combined to achieve a distinguisher for the compression function with complexity $2^{114}$. In [13] Chen et al. proposed related-key boomerang distinguishers on 32-step, 33-step and 34-step Threefish-512. Recently, Yu et al. [14] proposed boomerang attacks on the 32-step, 33-step and 34-step Skein-512.

Khovratovich et al. introduced rotational distinguishers in [15], where two words are said to be rotational if they are equal up to bit-wise rotation by some number of positions. Slide attacks were introduced by Biryukov et al. [16] and subsequently were applied to many cryptographic primitives.

Our Contribution. In the first part of this paper, we present a boomerang attack on the SM3 hash function reduced to 32 steps out of 64 steps with complexity $2^{14.4}$, 33 steps with complexity $2^{32.4}, 34$ steps with complexity $2^{53.1}$ and 35 steps with complexity $2^{117.1}$. Particular examples of the boomerang distinguisher for the 32-step and also the 33 -step compression function are provided. The previous results and a summary of ours are given in Table 1

In the second part of the paper, we present a slide-rotational property of SM3 and we analyze the SM3-XOR compression function, which is the SM3 compression function with the addition mod $2^{32}$ replaced by XOR. In particular, we show that, for SM3-XOR, one can easily construct input-output pairs satisfying a simple rotational property. Such a property exists due to the fact that, unlike in SHA-2, the constants in steps $i, i+1$, for $i=0, \ldots, 63, i \neq 15$ are computed by bitwise rotation starting from two predefined independent values. Previously, SHA2-XOR was analyzed in [17].

Paper Outline. The rest of the paper is organized as follows. In Section 2, we briefly review the specifications of the SM3 hash function and give the notation used in this paper. A brief overview of boomerang attacks is provided in Section 3 . The differential characteristics, and a description of the boomerang attack process and its complexity evaluation are provided in Section 4 The slide-rotational property is explained in Section 5 Finally, our conclusion is given in Section 6 . 
Table 1. Summary of the attacks on the SM3 compression function (CF) and hash function (HF)

\begin{tabular}{|c|c|c|c|c|}
\hline Attack & CF/HF & Steps & Complexity & Reference \\
\hline Preimage attack & HF & 28 & $2^{241.5}$ & {$[2]$} \\
\hline Preimage attack & HF & 30 & $2^{249}$ & {$[2]$} \\
\hline Boomerang attack & CF & 32 & $2^{14.4}$ & Section 4 \\
\hline Boomerang attack & CF & 33 & $2^{32.4}$ & Section 4 \\
\hline Boomerang attack & CF & 34 & $2^{53.1}$ & Section 4 \\
\hline Boomerang attack & CF & 35 & $2^{117.1}$ & Section 4 \\
\hline
\end{tabular}

\section{Description of SM3 and Notation}

In this section, we briefly review relevant specifications of the SM3 hash function and provide the notation used throughout the paper.

\subsection{Description of SM3}

The SM3 hash function compresses messages of arbitrary length into 256-bit hash values. Given any message, the algorithm first pads it into a message of length that is a multiple of 512 bits. We omit the padding method here since it is irrelevant to our attack. For our purpose, SM3 consists mainly of two parts: the message expansion and the state update transformation. In here, we briefly review the relevant specifications of these two components. For a detailed description of the hash function, we refer the reader to [1].

Message Expansion. The message expansion of SM3 splits the 512-bit message block $M$ into 16 words $m_{i},(0 \leq i \leq 15)$, and expands them into 68 expanded message words $w_{i}(0 \leq i \leq 67)$ and 64 expanded message words $w_{i}{ }^{\prime}(0 \leq i \leq 63)$ as follows:

$$
\begin{aligned}
& w_{i}=\left\{\begin{array}{lr}
m_{i}, & 0 \leq i \leq 15, \\
P_{1}\left(w_{i-16} \oplus w_{i-9} \oplus\left(w_{i-3} \lll 15\right)\right) \oplus\left(w_{i-13} \lll 7\right) \oplus w_{i-6}, & 16 \leq i \leq 67,
\end{array}\right. \\
& w_{i}^{\prime}=w_{i} \oplus w_{i+4}, 0 \leq i \leq 63 .
\end{aligned}
$$

The functions $P_{0}(X)$ which is used in the state update transformation and $P_{1}(X)$ which is used in message expansion are given by

$$
\begin{aligned}
& P_{0}(X)=X \oplus(X \lll 9) \oplus(X \lll 17), \\
& P_{1}(X)=X \oplus(X \lll 15) \oplus(X \lll 23) .
\end{aligned}
$$

State Update Transformation. The state update transformation starts from an initial value $I V=\left(A_{0}, B_{0}, C_{0}, D_{0}, E_{0}, F_{0}, G_{0}, H_{0}\right)$ of eight 32 -bit words and updates them in 64 steps. In step $i+1(0 \leq i \leq 63)$ the 32-bit words $w_{i}$ and $w_{i}{ }^{\prime}$ are used to update the state variables $A_{i}, B_{i}, C_{i}, D_{i}, E_{i}, F_{i}, G_{i}, H_{i}$ as follows: 


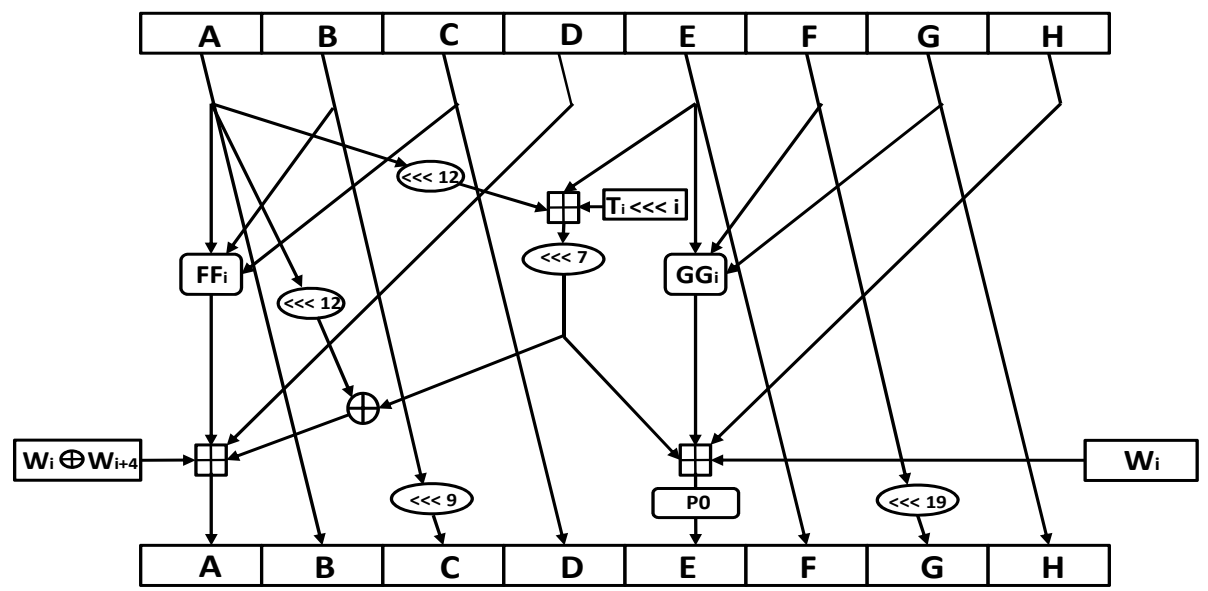

Fig. 1. One step of the SM3 hash function

$$
\begin{aligned}
S S 1_{i} & =\left(\left(A_{i} \lll 12\right)+E_{i}+\left(T_{i} \lll i\right)\right) \lll 7, \\
S S 2_{i} & =S S 1_{i} \oplus\left(A_{i} \lll 12\right), \\
T T 1_{i} & =F F_{i}\left(A_{i}, B_{i}, C_{i}\right)+D_{i}+S S 2_{i}+w_{i}{ }^{\prime}, \\
T T 2_{i} & =G G_{i}\left(E_{i}, F_{i}, G_{i}\right)+H_{i}+S S 1_{i}+w_{i}, \\
A_{i+1} & =T T 1_{i}, B_{i+1}=A_{i}, C_{i+1}=\left(B_{i} \lll 9\right), D_{i+1}=C_{i}, \\
E_{i+1} & =P_{0}\left(T T 2_{i}\right), F_{i+1}=E_{i}, G_{i+1}=\left(F_{i} \lll 19\right), H_{i+1}=G_{i} .
\end{aligned}
$$

The round constants are $T_{i}=0 \times 79 c c 4519$ for $i \in\{0, \ldots, 15\}$ and $T_{i}=0 x 7 a 879 d 8 a$, for $i \in\{16, \ldots, 63\}$. As for the bitwise Boolean functions $F F(X, Y, Z)$ and $G G(X, Y, Z)$ used in each step, we have

$$
\begin{aligned}
& F F(X, Y, Z)=\left\{\begin{array}{lr}
X \oplus Y \oplus Z, & 0 \leq i \leq 15, \\
(X \wedge Y) \vee(X \wedge Z) \vee(Y \wedge Z), & 16 \leq i \leq 63,
\end{array}\right. \\
& G G(X, Y, Z)=\left\{\begin{array}{lr}
X \oplus Y \oplus Z, & 0 \leq i \leq 15, \\
(X \wedge Y) \vee(\neg X \wedge Z), & 16 \leq i \leq 63
\end{array}\right.
\end{aligned}
$$

If $M$ is the last block, then $\left(A_{64} \oplus A_{0}, B_{64} \oplus B_{0}, C_{64} \oplus C_{0}, D_{64} \oplus D_{0}, E_{64} \oplus D_{0}, F_{64} \oplus\right.$ $\left.F_{0}, G_{64} \oplus G_{0}, H_{64} \oplus H_{0}\right)$ is the hash value. Otherwise $\left(A_{64} \oplus A_{0}, B_{64} \oplus B_{0}, C_{64} \oplus C_{0}, D_{64} \oplus D_{0}\right.$, $\left.E_{64} \oplus D_{0}, F_{64} \oplus F_{0}, G_{64} \oplus G_{0}, H_{64} \oplus H_{0}\right)$ constitutes the input of the next message block. One step of the SM3 compression function is depicted in Fig. 1

\subsection{Notation}

Our attacks use the integer modular subtraction difference. In here, we introduce the notation used in throughout the rest of the paper. 
1. $X, Y, X^{\prime}$ and $Y^{\prime}$ represent four 256-bit middle chaining values.

2. $I V_{X}, I V_{Y}, I V_{X^{\prime}}$ and $I V_{Y^{\prime}}$ represent four 256-bit initial values.

3. $H_{X}, H_{Y}, H_{X^{\prime}}$ and $H_{Y^{\prime}}$ represent four 256-bit outputs of the compression function.

4. $M_{X}, M_{Y}, M_{X^{\prime}}$ and $M_{Y^{\prime}}$ represent four 512-bit message blocks.

5. $w_{i, j}$ denotes the $j$-th bit of $w_{i}, 0 \leq j \leq 31$.

6. $\left(W_{t}\right)_{i}^{j}\left(t\right.$ can be $X, Y, X^{\prime}$ or $\left.Y^{\prime}\right)$ denotes $(j-i+1) 32$-bit words, $w_{i}$ to $w_{j}$, where $i<j$.

7. $S_{i}[+j]=S_{i}+2^{j}$ with no bit carry, $S_{i}[-j]=S_{i}-2^{j}$ with no bit carry, where $S$ can be $w, A, B, C, D, E, F, G, H$.

8. $S_{i}^{M_{c}}$ denotes the chaining value used in step $i$ combined with the middle chaining value $M_{c}$ where $M_{c}$ can be $X, Y, X^{\prime}, Y^{\prime}$, and $S$ can be $w, w^{\prime}, A, B, C, D, E, F, G, H, S S 1$, or $S S 2$.

\section{Boomerang Distinguishers for Hash Functions}

In this section, we review known-related-key boomerang attacks which can be used to distinguish a given permutation from a random oracle. We concentrate on the knownrelated-key boomerang attack to the compression function in the Davies-Mayer mode, i.e., $C F(M, K)=E(M, K) \oplus M$. As noted in [5|7|9]14], we can start from middle steps to construct boomerang distinguishers. Then we have

$$
C F_{0}^{-1}\left(X, K_{1}\right) \oplus C F_{0}^{-1}\left(X \oplus \beta, K_{2}\right)=\alpha,
$$

and

$$
C F_{1}\left(X, K_{1}\right) \oplus C F_{1}\left(X \oplus \gamma, K_{3}\right)=\delta,
$$

where the differential in $C F_{0}^{-1}$ holds with probability $p_{0}$ and holds with probability $p_{1}$ in $C F_{1}$. Using these two differentials, we can construct the boomerang attack for the compression function $C F$ as follows:

1. Choose a random value $X$, compute the corresponding value $X^{\prime}=X \oplus \beta, Y=$ $X \oplus \gamma, Y^{\prime}=Y \oplus \beta$ and $K_{2}=K_{1} \oplus \beta_{k}, K_{3}=K_{1} \oplus \gamma_{k}, K_{4}=K_{3} \oplus \beta_{k}$.

2. Compute backward from $\left(X, K_{1}\right),\left(X^{\prime}, K_{2}\right),\left(Y, K_{3}\right),\left(Y^{\prime}, K_{4}\right)$ using $C F_{0}^{-1}$ to obtain $P, P^{\prime}, Q, Q^{\prime}$.

3. Compute forward from $\left(X, K_{1}\right),\left(X^{\prime}, K_{2}\right),\left(Y, K_{3}\right),\left(Y^{\prime}, K_{4}\right)$ using $C F_{1}$ to obtain $C, C^{\prime}$, $D, D^{\prime}$.

4. Check whether $P \oplus P^{\prime}=Q \oplus Q^{\prime}=\alpha$ and $C \oplus D=C^{\prime} \oplus D^{\prime}=\delta$.

From (1) and (2),

$$
P \oplus P^{\prime}=Q \oplus Q^{\prime}=\alpha \text { and } C \oplus D=C^{\prime} \oplus D^{\prime}=\delta,
$$

holds with probability at least $p_{0}^{2}$ in the backward direction and with probability at least $p_{1}^{2}$ in the forward direction. Hence, assuming that the differentials are independent, the attack succeeds with probability $p_{0}^{2} p_{1}^{2}$. The expected number of solutions to (3) is 1 , if we repeat the attack about $1 /\left(p_{0}^{2} p_{1}^{2}\right)$ times.

For an n-bit random permutation, there exist 3 types of boomerang distinguishers which are summarized by $\mathrm{Yu}$ et al. in [14]. Here we recall the three distinguishers as follows. 
- Type I: A quartet that satisfies $P \oplus P^{\prime}=Q \oplus Q^{\prime}=\alpha$, and $C \oplus D=C^{\prime} \oplus D^{\prime}=\delta$ where the differences $\alpha$ and $\delta$ are fixed. In this case, there exists a generic attack with complexity $2^{n}$.

- Type II: A quartet that only satisfies the condition $C \oplus D=C^{\prime} \oplus D^{\prime}$. This type of attack is called second-order differential collision attack or zero-sum attack. In this case, we can use Wagner's generalized birthday attack [19] to obtain a quartet with the complexity $2^{n / 3}$.

- Type III: A quartet satisfies the conditions $P \oplus P^{\prime}=Q \oplus Q^{\prime}$ and $C \oplus D=C^{\prime} \oplus D^{\prime}$. The complexity of this attack is about $2^{n / 2}$.

In this paper, we apply a type III attack to develop distinguishers for 32/33/34/35 steps of the SM3 compression function. Therefore, the attack is valid if $p_{0}^{2} \cdot p_{1}^{2}>2^{-n / 2}$.

\section{Attacks on the SM3 Compression Function}

In this section, we describe the proposed boomerang attack on the SM3 compression function reduced to 32 steps, and then expend our attack to 33, 34 and 35 steps. Firstly, we give a summary of the differential characteristics to be used to distinguish the target compression function from random functions. Secondly, we describe how to use the message modification technique to correct the conditions in the intermediate steps by modifying the chaining values $A_{16}$ to $H_{16}$. We express our attack algorithm on 32step SM3 compression function in the third part of this section. Then we evaluate the complexity of our attack and extend it to 33,34 and 35 steps.

\subsection{Differential Characteristics}

In here, we mainly describe the differential characteristics which are used to attack 32step SM3 compression function. In Table 2, we present a differential characteristic in the backward direction from step 16 to step 1 which holds with probability $2^{-67}$, and the sufficient conditions that ensure that this characteristic holds. A differential characteristic in the forward direction from step 17 to step 32 which holds with probability $2^{-34}$ and its associated sufficient conditions are presented in Table 3

Finding the differential characteristics for both backward and forward directions is an important part of the attack. We construct the differential characteristics as follows.

- The characteristic has a single bit difference in the message word $w_{i}$ at some step, $i$, followed by 15 message words without differences. When using such characteristic, 12 steps (the ones that follow $i$ ) can be bypassed with probability 1 . Because of the fast diffusion of the difference coming from the message words, any characteristic that does not follow this strategy will have a low probability.

- In the backward direction, the differences in the message words are chosen as follows: $\Delta w_{2}=[+31], \Delta w_{i}=0(0 \leq i \leq 15, i \neq 2)$. Because $\Delta w_{2}=[+31]$ and $w_{2}{ }^{\prime}=w_{2} \oplus w_{6}$, we choose $w_{6,31}=0$ to ensure that $\Delta w_{2}{ }^{\prime}=[+31]$. Since $w_{18}=$ $P_{1}\left(w_{2} \oplus w_{9} \oplus\left(w_{15} \lll 15\right)\right) \oplus\left(w_{5} \lll 7\right) \oplus w_{12}$, by choosing proper $w_{12,14}, w_{12,22}$ and $w_{12,31}$, we can ensure that $\Delta w_{18}=[+14,+22,+31]$ holds. Combined with $w_{14, i}=0$ 
Table 2. Differential characteristic for steps 1-16 using signed bit-wise differences (32 steps)

\begin{tabular}{|c|c|c|c|c|c|}
\hline Step & $\begin{array}{l}\text { Differences of } \\
\text { chaining values }\end{array}$ & $\mathrm{w}$ & $w^{\prime}$ & $\operatorname{Pr}$ & Sufficient conditions \\
\hline & $\begin{array}{l}B_{0}:[+22] \\
C_{0}:[-31] \\
D_{0}:[-22,+31] \\
F_{0}:[+12] \\
G_{0}:[-31] \\
H_{0}:[-12,+31]\end{array}$ & & & $2^{-6}$ & $\begin{array}{l}A_{0,22}=C_{0,22}, \\
E_{0,12}=G_{0,12} \\
D_{0,22}=1, D_{0,31}=0, \\
H_{0, i}=1(i=12,31)\end{array}$ \\
\hline 1 & $\begin{array}{l}C_{1}:[+31] \\
D_{1}:[-31] \\
G_{1}:[+31] \\
H_{1}:[-31]\end{array}$ & & & $2^{-2}$ & $\begin{array}{l}D_{1,31}=1, \\
H_{1,31}=1 .\end{array}$ \\
\hline 2 & $\begin{array}{l}D_{2}:[+31] \\
H_{2}:[+31]\end{array}$ & {$[+31]$} & {$[+31]$} & $2^{-2}$ & $\begin{array}{l}D_{2,31}=0, \\
H_{2,31}=0 .\end{array}$ \\
\hline 3 & & & & 1 & \\
\hline$\vdots$ & : & : & $\vdots$ & : & : \\
\hline 14 & & & {$[+14,+22,+31]$} & 1 & \\
\hline 15 & $A_{15}:[+14,+22,+31]$ & & & $2^{-57}$ & $\begin{array}{l}A_{16, i}=0(i=1,2,9,11,14,18,22,26,31), \\
B_{16, i}=0(i=6,14,21,22,29,31), \\
C_{16,8}=D_{16,31}, C_{16,23}=D_{16,14}, \\
C_{16,31}=D_{16,22}, \\
\left(B_{16} \lll 12\right)+F_{16}=-\left(T_{15} \lll 15\right), \\
E_{16, i}=0(i=1,3,9,10,18,26,27), \\
\bigoplus_{i \in \Lambda_{j}} E_{16, i}=0, j \in\{1,2,3\}, \\
\Lambda_{1}=\{6,14,15,16,22,24,30,31\}, \\
\Lambda_{2}=\{0,6,7,14,22,23,24,30\}, \\
\Lambda_{3}=\{0,7,15,16,23,31\} .\end{array}$ \\
\hline 16 & $\begin{aligned} A_{16}: & {[+1,+2,+9,+11,} \\
& +14,+18,+22, \\
& +26,+31] \\
B_{16} & :[+14,+22,+31] \\
E_{16} & :[+1,+3,+9,+10 \\
& +18,+26,+27]\end{aligned}$ & & & & \\
\hline
\end{tabular}

$(i=14,22,31)$, we can get $\Delta w_{14}{ }^{\prime}=[+14,+22,+31]$. So the differences of the message words in the backward direction are $\Delta w_{2}=[+31], \Delta w_{2}^{\prime}=[+31], \Delta w_{14}{ }^{\prime}=$ $[+14,+22,+31]$, and all the other message words differences are zero.

If $A_{15}[+14,+22,+31]$ holds, then we can cancel the differences $\Delta w_{14}{ }^{\prime}=[+14$, $+22,+31$ ] in step 15 , and skip 12 steps from step 15 to step 4 with probability 1 . The following is the derivation for the sufficient conditions in step 16 of Table 2 . The differential characteristic in step 16 is given by: 
Table 3. Differential characteristic for steps 17-32 using signed bit-wise differences

\begin{tabular}{|c|c|c|c|c|c|}
\hline Step & $\begin{array}{l}\text { Differences of } \\
\text { chaining values }\end{array}$ & $\mathrm{w}$ & $w^{\prime}$ & $\operatorname{Pr}$ & Sufficient conditions \\
\hline 16 & $\begin{aligned} A_{16}:[-6] \\
D_{16}:[-6,-11,-13, \\
\quad-18,-19,-20, \\
\quad-22,-25,-26] \\
E_{16}:[-28] \\
H_{16}:[-4,-5,-11, \\
\quad-13,-19,-20, \\
-22,-25,-26]\end{aligned}$ & $\begin{array}{l}{[+3,+4,+5,} \\
+11,+13, \\
+19,+20, \\
+22,+27]\end{array}$ & $\begin{array}{l}{[+3,+4,+5,} \\
+11,+13, \\
+19,+20, \\
+22,+27]\end{array}$ & & \\
\hline 17 & $\begin{array}{l}B_{17}:[-6] \\
F_{17}:[-28]\end{array}$ & & & $2^{-27}$ & $\begin{array}{l}A_{16, i}=1(i=6,23), A_{16,13}=0 \\
B_{16,6}=C_{16,6} \\
D_{16, i}=1(i=6,11,13,18,19,20,22,25,26) \\
E_{16,28}=1, F_{16,28}=G_{16,28} \\
H_{16, i}=1(i=4,5,11,13,19,20,22,25,26) \\
S S 1_{16, i}=1(i=3,18,25)\end{array}$ \\
\hline 18 & $\begin{array}{l}C_{18}:[-15] \\
G_{18}:[-15]\end{array}$ & & & $2^{-2}$ & $\begin{array}{l}A_{17,6}=C_{17,6} \\
E_{17,28}=0\end{array}$ \\
\hline 19 & $\begin{array}{l}D_{19}:[-15] \\
H_{19}:[-15]\end{array}$ & {$[+15]$} & {$[+15]$} & $2^{-2}$ & $\begin{array}{l}A_{18,15}=B_{18,15} \\
E_{18,15}=1\end{array}$ \\
\hline 20 & & & & 1 & \\
\hline$\vdots$ & : & & : & $\vdots$ & $\vdots$ \\
\hline 31 & & & {$[ \pm 6,+15,+30]^{1}$} & 1 & \\
\hline 32 & $A_{32}:[ \pm 6,+15,+30]$ & & & $2^{-3}$ & \\
\hline
\end{tabular}

${ }^{1}$ The difference of $w_{2}$ affects $w_{31,6}$. In other words, if we choose $w_{31,6}^{X}-w_{31,6}^{Y}=+1$, then the difference $w_{31,6}^{X^{\prime}}-w_{31,6}^{Y^{\prime}}=-1$, and vice versa (We used " \pm " to denote this fact). Note that this does not affect the XOR-differences in step-32.

$$
\begin{gathered}
\left(A_{16}[+1,+2,+9,+11,+14,+18,+22,+26,+31], B_{16}[+14,+22,+31], C_{16}, D_{16},\right. \\
\left.E_{16}[+1,+3,+9,+10,+18,+26,+27], F_{16}, G_{16}, H_{16}\right) \longrightarrow \\
\left(A_{15}[+14,+22,+31], B_{15}, C_{15}, D_{15}, E_{15}, F_{15}, G_{15}, H_{15}\right) .
\end{gathered}
$$

1. Because $A_{15}=B_{16}, B_{15}=\left(C_{16} \ggg 9\right), C_{15}=D_{16}, E_{15}=F_{16}, F_{15}=\left(G_{16} \ggg\right.$ $19)$ and $G_{15}=H_{16}$, we choose $\left(B_{16} \lll 12\right)+F_{16}=-\left(T_{15} \lll 15\right)$ to ensure that $\Delta S S 1=\Delta\left(\left(B_{16} \lll 12\right)+F_{16}+\left(T_{15} \lll 15\right)\right) \lll 7=[+1,+9,+18]$ holds and the conditions $B_{16, i}=0(i=6,21,29)$ ensure that $\Delta S S 2=\Delta S S 1 \oplus \Delta\left(B_{16} \lll\right.$ $12)=[+1,+2,+9,+11,+18,+26]$ holds.

2. The conditions $B_{15, i}=C_{15, i},(i=14,22,31)$, i.e., $C_{16,8}=D_{16,31}, C_{16,23}=D_{16,14}$ and $C_{16,31}=D_{16,22}$ ensure that $\Delta F F_{15}\left(A_{15}, B_{15}, C_{15}\right)=\Delta F F_{15}\left(B_{16}, C_{16} \ggg 9\right.$, $\left.F_{16}\right)=[+14,+22,+31]$ hold. Combined with $\Delta w_{15}{ }^{\prime}=0$, we can get $\Delta D_{15}=$ $\Delta A_{16}-\left(\Delta F F_{15}\left(B_{16}, C_{16} \ggg 9, D_{16}\right)+\Delta S S 2+\Delta w_{15}{ }^{\prime}\right)=0$. Similarly, the conditions $\bigoplus_{i \in \Lambda_{j}} E_{16, i}=0, j \in\{1,2,3\}, \Lambda_{1}=\{6,14,15,16,22,24,30,31\}$, $\Lambda_{2}=\{0,6,7,14,22,23,24,30\}, \Lambda_{3}=\{0,7,15,16,23,31\}$ ensure that $\Delta H_{15}=0$ holds.

Thus the above conditions constitute a set of sufficient conditions for the differential characteristic in step 16 . 
Table 4. Differential characteristic for steps 1-16 using signed bit-wise differences (33 steps)

\begin{tabular}{|c|c|c|c|c|c|}
\hline Step & $\begin{array}{l}\text { Differences of } \\
\text { chaining values }\end{array}$ & $\mathrm{w}$ & $w^{\prime}$ & $\operatorname{Pr}$ & Sufficient conditions \\
\hline & $\begin{array}{l}B_{0}:[+22] \\
C_{0}:[-31] \\
D_{0}:[-22,+31] \\
F_{0}:[+12] \\
G_{0}:[-31] \\
H_{0}:[-12,+31]\end{array}$ & & & $2^{-6}$ & $\begin{array}{l}A_{0,22}=C_{0,22} \\
E_{0,12}=G_{0,12} \\
D_{0,22}=1, D_{0,31}=0 \\
H_{0, i}=1(i=12,31)\end{array}$ \\
\hline 1 & $\begin{array}{l}C_{1}:[+31] \\
D_{1}:[-31] \\
G_{1}:[+31] \\
H_{1}:[-31]\end{array}$ & & & $2^{-2}$ & $\begin{array}{l}D_{1,31}=1, \\
H_{1,31}=1 .\end{array}$ \\
\hline 2 & $\begin{array}{l}D_{2}:[+31] \\
H_{2}:[+31]\end{array}$ & {$[+31]$} & {$[+31]$} & $2^{-2}$ & $\begin{array}{l}D_{2,31}=0 \\
H_{2,31}=0 .\end{array}$ \\
\hline 3 & & & & 1 & \\
\hline$\vdots$ & : & : & : & : & : \\
\hline 14 & & & {$[+14,+22,+31]$} & 1 & \\
\hline 15 & $A_{15}:[+14,+22,+31]$ & & & $2^{-59}$ & $\begin{array}{l}A_{16, i}=0(i=3,9,11,14,15,16,17,22,26,31), \\
A_{16,1}=1, B_{16, i}=0(i=6,14,21,22,29,31), \\
C_{16,8}=D_{16,31}, C_{16,23} \neq D_{16,14}, C_{16,31}=D_{16,22}, \\
\left(B_{16} \lll 12\right)+F_{16}=-\left(T_{15} \lll 15\right), \\
E_{16, i}=0(i=1,3,9,10,18,26,27), \\
\bigoplus_{i \in \Lambda_{j}} E_{16, i}=0, j \in\{1,2,3\}, \\
\Lambda_{1}=\{6,14,15,16,22,24,30,31\}, \\
\Lambda_{2}=\{0,6,7,14,22,23,24,30\}, \\
\Lambda_{3}=\{0,7,15,16,23,31\} .\end{array}$ \\
\hline 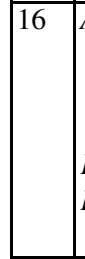 & $\begin{aligned} A_{16} & :[-1,+3,+9,+11, \\
& +14,+15,+16, \\
& +17,+22, \\
& +26,+31] \\
B_{16} & :[+14,+22,+31] \\
E_{16} & :[+1,+3,+9,+10 \\
& +18,+26,+27]\end{aligned}$ & & & & \\
\hline
\end{tabular}

- In the forward direction, we choose the message differences as follows: $\Delta w_{19}=$ $[+15], \Delta w_{i}=0(20 \leq i \leq 34)$. Since $w_{19}{ }^{\prime}=w_{19} \oplus w_{23}$, we choose $w_{23,15}=0$ to ensure that $\Delta w_{19^{\prime}}=[+15]$. Because $\Delta w_{19}=[+15]$ and $w_{19}=P_{1}\left(w_{3} \oplus w_{10}\right.$ $\left.\oplus\left(w_{16} \lll 15\right)\right) \oplus\left(w_{6} \lll 7\right) \oplus w_{13}$, let $w_{3} \oplus w_{10}=0$ and $\left(w_{6} \lll 7\right) \oplus w_{13}=0$, to get $\Delta w_{16}=[+3,+4,+5,+11,+13,+19,+20,+22,+27]$. If we choose $w_{20, i}=0$, $(i=3,4,5,11,13,19,20,22,27)$ and $w_{23,15}=0$, from $w_{16}{ }^{\prime}=w_{16} \oplus w_{20}$, we can get $\Delta w_{16}{ }^{\prime}=\Delta w_{16}$. Because $w_{35}=P_{1}\left(w_{19} \oplus w_{26} \oplus\left(w_{32} \lll 15\right)\right) \oplus\left(w_{22} \lll 7\right) \oplus w_{29}$ and $w_{31}{ }^{\prime}=w_{31} \oplus w_{35}$, we choose $w_{31, i}=0(i=6,15,30)$, such that $\Delta w_{31}{ }^{\prime}=$ $[ \pm 6,+15,+30]^{-1}$.

$\overline{{ }^{1} \text { Because } w_{31}}=P_{1}\left(w_{15} \oplus w_{22} \oplus\left(w_{28} \lll 15\right)\right) \oplus\left(w_{18} \lll 7\right) \oplus w_{25}$ and $\Delta w_{18}=[+14,+22,+31]$ in the backward direction, if we choose $w_{31, i}^{X}=0$ and $w_{31, i}^{Y}=1(i=6,15,30)$, then the bits in $w_{31}^{X^{\prime}}$ and $w_{31}^{Y^{\prime}}$ are $w_{31, i}^{X^{\prime}}=0(i=15,30), w_{31,6}^{X^{\prime}}=1$ and $w_{31, i}^{Y^{\prime}}=1(i=15,30), w_{31,6}^{Y^{\prime}}=0$. So 
Table 5. Differential characteristic for steps 17-33 using signed bit-wise differences

\begin{tabular}{|c|c|c|c|c|c|}
\hline Step & $\begin{array}{l}\text { Differences of } \\
\text { chaining values }\end{array}$ & $\mathrm{w}$ & $w^{\prime}$ & $\operatorname{Pr}$ & Sufficient conditions \\
\hline 16 & $\begin{array}{c}C_{16}:[-0,-2,-5,-6,-18, \\
-23,-25,-30,-31] \\
D_{16}:[-27] \\
G_{16}:[-0,-2,-5,-6,-16, \\
-17,-23,-25,-31] \\
H_{16}:[-3,-8,-10,-11, \\
-19,-26,-27]\end{array}$ & & {$[+27]$} & & \\
\hline 17 & \begin{tabular}{|r|}
$A_{17}:[-18]$ \\
$D_{17}:[-0,-2,-5,-6,-18$, \\
$-23,-25,-30,-31]$ \\
$E_{17}:[-8]$ \\
$H_{17}:[-0,-2,-5,-6,-16$, \\
$-17,-23,-25,-31]$
\end{tabular} & $\begin{array}{l}{[+0,+2,+7} \\
+15,+16 \\
+17,+23 \\
+25,+31]\end{array}$ & $\begin{array}{l}{[+0,+2,+7} \\
+15,+16 \\
+17,+23 \\
+25,+31]\end{array}$ & $2^{-54}$ & $\begin{array}{l}A_{16, i}=B_{16, i}(i=0,2,5,6,23,25,30,31) \\
A_{16,18}=B_{16,18} \\
C_{16, i}=1(i=0,2,5,6,18,23,25,30,31) \\
T T 1_{16,8}=0, D_{16,27}=1 \\
E_{16, i}=1(i=0,2,5,6,16,23,31) \\
E_{16, i}=0(i=17,25) \\
G_{16, i}=1(i=0,2,5,6,16,17,23,25,31) \\
H_{16, i}=1(i=3,8,10,11,19,26,27) \\
T T 2_{16, i}=1(i=3,8,10,11,17,19,25,26,27)\end{array}$ \\
\hline \begin{tabular}{|l|}
18 \\
18
\end{tabular} & $\begin{array}{l}B_{18}:[-18] \\
F_{18}:[-8]\end{array}$ & & & $2^{-9}$ & $\begin{array}{l}A_{17, i}=1(i=3,18), A_{17,25}=0 \\
B_{17,18}=C_{17,18} \\
E_{17,8}=1, F_{17,8}=G_{17,8} \\
S S 1_{17, i}=1(i=5,15,30)\end{array}$ \\
\hline 19 & \begin{tabular}{|l|}
$C_{19}:[-27]$ \\
$G_{19}:[-27]$ \\
\end{tabular} & & & $2^{-2}$ & $\begin{array}{l}A_{18,18}=C_{18,18} \\
E_{18,8}=0 \\
\end{array}$ \\
\hline 20 & $\begin{array}{l}D_{20}:[-27] \\
H_{20}:[-27] \\
\end{array}$ & {$[+27]$} & {$[+27]$} & $2^{-2}$ & $\begin{array}{l}A_{19,27}=B_{19,27} \\
E_{19,27}=1 .\end{array}$ \\
\hline 21 & & & & 1 & \\
\hline$\vdots$ & $\vdots$ & & & $\vdots$ & $\vdots$ \\
\hline 32 & & & {$[+10,+18,+27]$} & 1 & \\
\hline 33 & $A_{32}:[+10,+18,+27]$ & & & $2^{-3}$ & \\
\hline
\end{tabular}

In this case, the massage word differences in the forward direction are $\Delta w_{16}=$ $\Delta w_{16}{ }^{\prime}=[+3,+4,+5,+11,+13,+19,+20,+22,+27], \Delta w_{19}=\Delta w_{19}{ }^{\prime}=[+15], \Delta w_{31}{ }^{\prime}=$ $[ \pm 6,+15,+30]$, and all the other message words differences are zero.

The following is the derivation for the sufficient conditions for step 17 in Table 3 . The differential characteristic in step 17 is given by:

$$
\begin{gathered}
\left(A_{16}[-6], B_{16}, C_{16}, D_{16}[-6,-11,-13,-18,-19,-20,-22,-25,-26], E_{16}[-28], F_{16}\right. \\
\left.G_{16}[+28], H_{16}[-4,-5,-11,-13,-19,-20,-22,-25,-26]\right) \rightarrow \\
\left(A_{17}, B_{17}[-6], C_{17}, D_{17}, E_{17}, F_{17}[-28], G_{17}, H_{17}\right) .
\end{gathered}
$$

the difference of $w_{2}$ affects $w_{31,6}$. In other words, if we choose $w_{31,6}^{X}-w_{31,6}^{Y}=+1$, then the difference $w_{31,6}^{X^{\prime}}-w_{31,6}^{Y^{\prime}}=-1$, and vice versa (We used " \pm " to denote this fact). Note that this does not affect the XOR-differences in step 32. 
1. From $\Delta A_{16}=[-6]$ and $B_{16,6}=C_{16,6}$, we get $\Delta F F_{16}\left(A_{16}, B_{16}, C_{16}\right)=0$. From $\Delta E_{16}=[-28]$ and $F_{16,28}=G_{16,28}$, we get $\Delta G G_{16}\left(E_{16}, F_{16}, G_{16}\right)=0$.

2. From $\Delta A_{16}=[-6]$ and $\Delta E_{16}=[-28]$, it follows that the conditions $S S 1_{16,3}=$ $1, S S 1_{16,25}=1$ ensure $\Delta S S 1_{16}=[-3,-25]$. Combined with the conditions $S S 1_{16,18}=1, A_{16,13}=0$ and $A_{16,23}=1$, we can get $\Delta S S 2_{16}=[+3,+18,-25]$. Therefore, $\Delta A_{17}=\Delta T T 1_{16}=0$, and $\Delta E_{17}=\Delta P_{0}\left(T T 2_{16}\right)=0$.

Thus the above conditions constitute a set of sufficient conditions for the differential characteristic in step 17.

\subsection{Message Modification}

We use the message modification technique which has been introduced by Wang et al. [18] to improve the complexity of our attack. We can modify the chaining values $A_{16}$ to $H_{16}$ to ensure that almost all the conditions in $A_{i}$ to $H_{i}(\mathrm{i}=17,18,19)$ hold. For example, in the backward direction, we can modify $E_{16, i}(i=6,15)$ to make the sufficient conditions $\bigoplus_{i \in \Lambda_{j}} E_{16, i}=0, j \in\{1,2,3\}, \Lambda_{1}=\{6,14,15,16,22,24,30,31\}$, $\Lambda_{2}=\{0,6,7,14,22,23,24,30\}, \Lambda_{3}=\{0,7,15,16,23,31\}$ hold.

In Table 3, there are 31 sufficient conditions from step 17 to step 19 in each differential. We can correct all the sufficient conditions in one differential by using message modification techniques, and the sufficient conditions $S S 1_{16, i}=1(i=3,18,25)$, $A_{17,6}=C_{17,6}, E_{17,28}=0, A_{18,15}=B_{18,15}$ and $E_{18,15}=1$ are not corrected in another differential. So the probability of step 17 to step 19 can be improved from $2^{-2 \times 31}=$ $2^{-62}$ to $2^{-7}$. In Table 5, there are 63 sufficient conditions from step 17 to step 18 in each differential. We can correct all the sufficient conditions in one differential by using message modification techniques. However, the sufficient conditions $T T 1_{16,8}=0$, $T T 2_{16, i}=1(i=3,8,10,11,17,19,25,26,27), A_{17, i}=1(i=3,18), A_{17,25}=0, E_{17,8}=1$ and $S S 1_{17, i}=1(i=5,15,30)$ are not corrected in the other differential. So the probability of step 17 to step 18 can be improved from $2^{-2 \times 63}=2^{-126}$ to $2^{-17}$. Consequently, in this case, the probability of step 17 to step 20 can be improved from $2^{-2 \times 67}=2^{-134}$ to $2^{-17-2 \times 4}=2^{-25}$.

\subsection{Boomerang Attacks on the 32-Step SM3 Compression Function}

The attack algorithm on 32-step SM3 compression function can be summarized as follows.

1. Choose a random 512-bit message $M$ and expand it to 36 words. Set proper message words as in section 4.1 to ensure that $\Delta w_{2}=[+31], \Delta w_{2}{ }^{\prime}=[+31], \Delta w_{14}{ }^{\prime}=[+14$, $+22,+31]$ in the backward direction, and $\Delta w_{16}=\Delta w_{16}{ }^{\prime}=[+3,+4,+5,+11,+13$, $+19,+20,+22,+27], \Delta w_{19}=[+15], \Delta w_{19}{ }^{\prime}=[+15], \Delta w_{31}{ }^{\prime}=[ \pm 6,+21,+29]$ in the forward direction. Let $M_{X}=M, M_{X^{\prime}}=M \oplus \Delta w_{2}$. Expend the messages $M_{X}$ and $M_{X^{\prime}}$ to 36 words $W_{X}$ and $W_{X^{\prime}}$, respectively. Let $W_{Y}=W_{X} \oplus \Delta w_{19}$ and $W_{Y^{\prime}}=$ $W_{X^{\prime}} \oplus \Delta w_{19}$. Then we use the 16 words $\left(W_{Y}\right)_{19}^{34}$ and $\left(W_{Y^{\prime}}\right)_{19}^{34}$ to get two 36-word $\left(W_{Y}\right)_{0}^{35}$ and $\left(W_{Y^{\prime}}\right)_{0}^{35}$ by using the message expansion algorithm. Let $M_{Y}=\left(W_{Y}\right)_{0}^{15}$, $M_{Y^{\prime}}=\left(W_{Y^{\prime}}\right)_{0}^{15}$. 
Table 6. Example of a quartet for 32 steps of the SM3 compression function

\begin{tabular}{|c|c|c|c|c|c|c|c|c|}
\hline \multicolumn{9}{|c|}{ Message } \\
\hline$\overline{M_{X}}$ & $\begin{array}{l}\text { ecffec51 } \\
6 \mathrm{a} 506 \mathrm{f} 8\end{array}$ & $\begin{array}{l}192 \mathrm{fa6b} 4 \\
33169484\end{array}$ & $\begin{array}{l}6314 \mathrm{~d} 06 \mathrm{c} \\
355 \mathrm{~b} 11 \mathrm{c} 5\end{array}$ & $\begin{array}{l}\text { f86f5604 } \\
6 \mathrm{~b} 81 \mathrm{ddd} 0\end{array}$ & $\begin{array}{l}\text { ed6f140d } \\
\text { le58820e }\end{array}$ & $\begin{array}{l}4597860 \mathrm{c} \\
78 \mathrm{fa} 46 \mathrm{f} 6\end{array}$ & $\begin{array}{l}3 \mathrm{a} 4 \operatorname{ccc} 9 a \\
742 \mathrm{~b} 217 \mathrm{f}\end{array}$ & $\begin{array}{l}\mathrm{b} 78 \mathrm{~b} 2 \mathrm{ded} \\
4669940 \mathrm{f}\end{array}$ \\
\hline \multirow[t]{2}{*}{$M_{X^{\prime}}$} & ecffec51 & $192 \mathrm{fa} 6 \mathrm{~b} 4$ & e314d06c & f86f5604 & ed6f140d & $4597860 \mathrm{c}$ & $3 a 4 \operatorname{ccc} 9 a$ & b78b2ded \\
\hline & $6 a 5 b 06 f 8$ & 33169484 & $355 \mathrm{~b} 11 \mathrm{c} 5$ & $6 \mathrm{~b} 81 \mathrm{ddd} 0$ & $1 \mathrm{e} 58820 \mathrm{e}$ & $78 \mathrm{fa} 46 \mathrm{f} 6$ & $742 \mathrm{~b} 217 \mathrm{f}$ & $4669940 \mathrm{f}$ \\
\hline$\overline{M_{Y}}$ & \begin{tabular}{|l|} 
e8fff051 \\
6a5b06f8
\end{tabular} & $\begin{array}{l}\text { lbafa634 } \\
430624 d 4\end{array}$ & $\begin{array}{l}6 \mathrm{~b} 4 \mathrm{cf} 854 \\
3 \mathrm{~d} 5311 \mathrm{~d} 5\end{array}$ & $\begin{array}{l}\text { a86ff654 } \\
\text { 6b81ddd0 }\end{array}$ & $\begin{array}{l}\text { 6dea968e } \\
\text { le58020e }\end{array}$ & $\begin{array}{l}4597060 \mathrm{c} \\
50 \mathrm{fa} 4 \mathrm{ef6} 6\end{array}$ & $\begin{array}{l}524 \mathrm{cc} 49 \mathrm{a} \\
742 \mathrm{~b} 217 \mathrm{f}\end{array}$ & $\begin{array}{l}\mathrm{b} 78 \mathrm{~b} 25 \mathrm{ed} \\
4669940 \mathrm{f}\end{array}$ \\
\hline$M_{Y^{\prime}}$ & $\begin{array}{l}\text { e8fff051 } \\
\text { 6a5b06f8 }\end{array}$ & $\begin{array}{l}\text { lbafa634 } \\
430624 d 4\end{array}$ & $\begin{array}{l}\text { eb4cf854 } \\
3 \mathrm{~d} 5311 \mathrm{~d} 5\end{array}$ & $\begin{array}{l}\text { a86ff654 } \\
6 \mathrm{~b} 81 \mathrm{ddd} 0\end{array}$ & $\begin{array}{l}\text { 6dea968e } \\
1 \mathrm{e} 58020 \mathrm{e}\end{array}$ & $\begin{array}{l}4597060 \mathrm{c} \\
50 \mathrm{fa} 4 \mathrm{ef6}\end{array}$ & $\begin{array}{l}524 \mathrm{cc} 49 \mathrm{a} \\
742 \mathrm{~b} 217 \mathrm{f}\end{array}$ & $\begin{array}{l}\text { b78b25ed } \\
4669940 f\end{array}$ \\
\hline \multicolumn{9}{|c|}{ Chaining Value } \\
\hline$I V_{X}$ & 0d548434 & 6f039a92 & $3 \mathrm{~d} 5 \mathrm{fb} 868$ & $01 \mathrm{~b} 03347$ & $29 \mathrm{c} 6 \mathrm{a} 571$ & 0d8b6217 & $4 \mathrm{f} 2359 \mathrm{fa}$ & d6a363f4 \\
\hline$I V_{X^{\prime}}$ & 0 0d548434 & $6 f 439 a 92$ & bd5fb868 & 81703347 & $29 \mathrm{c} 6 \mathrm{a} 571$ & $0 \mathrm{~d} 8 \mathrm{~b} 7217$ & cf2359fa & $56 \mathrm{a} 373 \mathrm{f4} 4$ \\
\hline$I V_{Y}$ & $792457 \mathrm{dc}$ & $8 \mathrm{f} 057732$ & $6137 \mathrm{fcd} 4$ & $7899 \mathrm{~b} 663$ & $948 \mathrm{~b} 29 \mathrm{bf}$ & d5f5a832 & $\begin{array}{l}\text { d9ae3751 } \\
\text { (3) }\end{array}$ & c747e405 \\
\hline$\overline{I V} V_{Y^{\prime}}$ & $792457 \mathrm{dc}$ & $8 \mathrm{f} 457732$ & $\mathrm{e} 137 \mathrm{fcd} 4$ & f859b663 & $948 \mathrm{~b} 29 \mathrm{bf}$ & d5f5b832 & $59 \mathrm{ae} 3751$ & $4747 \mathrm{f} 405$ \\
\hline$H_{X}$ & ale82b03 & $54 \mathrm{~b} 1 \mathrm{bb} 42$ & $2563 \mathrm{~b} 063$ & e514d921 & a0eaflfa & 632d0eef & e2a999cf & ad4964d1 \\
\hline$\overline{H_{X^{\prime}}}$ & $3 \mathrm{fd} 356 \mathrm{c} 7$ & ca7f9b81 & $3 \mathrm{a} 9694 \mathrm{~d} 6$ & $31 \mathrm{~d} 02769$ & b454a3bd & $\mathrm{c} 2 \mathrm{~d} 2 \mathrm{dc} 37$ & $45 \mathrm{ffc} 720$ & $2 \mathrm{e} 319 \mathrm{c} 71$ \\
\hline$\overline{H_{Y}}$ & ele8ab43 & $54 \mathrm{~b} 1 \mathrm{bb} 42$ & $2563 \mathrm{~b} 063$ & e514d921 & a0eaflfa & 632d0eef & e2a999cf & ad4964d1 \\
\hline$\overline{H_{Y^{\prime}}}$ & $7 \mathrm{fd} 3 \mathrm{~d} 687$ & ca7f9b81 & 3a9694d6 & $31 \mathrm{~d} 02769$ & b454a3bd & c2d2dc37 & $45 \mathrm{ffc} 720$ & $2 \mathrm{e} 319 \mathrm{c} 71$ \\
\hline
\end{tabular}

2. Randomly choose the chaining values $A_{16}, B_{16}, C_{16}, D_{16}, E_{16}, G_{16}$ and $H_{16}$ such that almost all the conditions used in step 16 and step 172 in Table 2 and Table 3 hold.

3. By using the message modification technique, modify $A_{16,19}, H_{16,28}, C_{16,15}$ and $G_{16,15}$ to make the sufficient conditions $A_{17,6}=C_{17,6}, E_{17,28}=0, A_{18,15}=B_{18,15}$ and $E_{18,15}=1$ hold in one of the differentials in the forward direction.

4. Use state update transformation process to get $I V_{X}, I V_{Y}, I V_{X^{\prime}}, I V_{Y^{\prime}}, H_{X}, H_{Y}, H_{X^{\prime}}$ and $H_{Y^{\prime}}$. Check whether $I V_{X} \oplus I V_{X^{\prime}}=I V_{Y} \oplus I V_{Y^{\prime}}$ and $H_{X} \oplus H_{Y}=H_{X^{\prime}} \oplus H_{Y^{\prime}}$ hold.

5. If a quartet is found, then a distinguisher is found. Repeat the above 4 steps with different messages and chaining values $\left(A_{16}\right.$ to $\left.H_{16}\right)$ until a distinguisher is found.

\subsection{Complexity of the Attack}

Using the differential characteristics and the message modification technique, we can construct the boomerang attack for the SM3 compression function reduced to 32 steps.

In the backward direction, all the sufficient conditions used in step 16 can be set in both of the differentials and the sufficient conditions used in step 3 to step 1 cannot be corrected in both of the differentials. So the differential characteristic used in the backward direction holds with probability $2^{-10}$. Thus both of the differentials used in the backward direction hold with probability $2^{-10 \times 2}=2^{-20}$. In the forward direction, 7 sufficient conditions, from step 17 to step 19, are not corrected in one of the differentials by using message modifications and in step 32, non of the sufficient conditions is corrected in both of the differentials. Thus both of the differentials used in the forward direction hold with probability $2^{-7-3 \times 2}=2^{-13}$ after the message modification.

Hence, we can give a boomerang attack on 32-step SM3 with complexity $2^{20+13}=$ $2^{33}$. We can also use the amplified differential characteristics to improve the complexity of the attack. In this case, both of the two differentials used in the backward direction hold with probability $2^{-3.2}$ and the two differentials used in step 32 hold with probability

\footnotetext{
${ }^{2}$ All the conditions used in step 16 can hold in both of the differentials. In step 17 the conditions $S S 1_{16, i}=1(i=3,18,25)$ cannot be corrected in one of the differentials and all the other conditions can hold.
} 
$2^{-4.2}$. So we can get a 32 -step boomerang distinguisher with complexity $2^{3.2+7+4.2}=$ $2^{14.4}$. An example of a 32-step boomerang distinguisher (quartet) is presented in Table 6 .

\subsection{Attacks on 33/34/35 Steps SM3 Compression Function}

In what follows we extend the proposed boomerang distinguisher to the 35-step SM3 compression function. First, we obtain a new 33-step boomerang distinguisher and then extend it to 35 steps. If we simply add one step in the forward differential characteristic in Table 3, this will result in some contradictions in $A_{16}$ and $E_{16}$ between Table 2 and Table 3. So we choose the message words differences as follows: $\Delta w_{2}=[+31], \Delta w_{i}=0$ $(0 \leq i \leq 15, i \neq 2)$ in the backward direction, and $\Delta w_{20}=[+27], \Delta w_{i}=0(21 \leq i \leq 35)$ in the forward direction.

We also correct $\Delta A_{16}=[-1,+3,+9,+11,+14,+15,+16,+17,+22,+26,+31]$ and change one of the sufficient conditions $C_{16,23}=D_{16,14}$ to $C_{16,23} \neq D_{16,14}$.

In this case, the backward direction is from step 16 to step 1 and the differential characteristic which is given in Table 4 holds with probability $2^{-3.2}$. The forward direction is from step 17 to step 33 and the differential characteristic is given in Table 5 where 25 sufficient conditions are not corrected in one of the differentials by using message modifications. So the forward differential characteristic holds with probability $2^{-25-4.2}=2^{-29.2}$. So we can get the 33-step boomerang distinguisher with complexity $2^{3.2+29.2}=2^{32.4}$. In step 34 both of the differentials hold with probability $2^{-20.7}$ using the amplified differential characteristics. Thus we obtain a 34-step boomerang distinguisher with complexity $2^{32.4+20.7}=2^{53.1}$. We also assume $A_{35}$ and $E_{35}$ all have 32-bit differences. Thus the 35 -step boomerang distinguisher has a complexity $\approx 2^{53.1+2 \times 32}=2^{117.1}$.

\section{A Slide-Rotational Property of SM3-XOR}

In this section, we show that, in the case of the full SM3-XOR, pairs satisfying a certain rotational relation can be easily generated. An example of such a pair for the SM3-XOR is provided in Table 8 . Such a property is not known to exist for SHA2-XOR [17].

The above mentioned property exists due to the fact that the constants over the 64 steps of SM3 are related. According to the SM3 specification, in steps $j \in\{0, \ldots, 15\}$, one constant rotated by $j$ is utilized, whereas the other constant rotated by $j$ is used in steps $j \in\{16, \ldots, 63\}$. Since operations like XOR, $F F_{i}, G G_{i}, 0 \leq i<64$, that are used in the SM3-XOR step function preserve the rotational property, it is natural to attempt a rotational attack, as provided below. We note that if instead of SM3-XOR, the original SM3 compression function is used, the addition mod $2^{32}$ transforms the attack into a probabilistic one, as outlined below. Due to the high number of additions per step, it appears difficult to exploit this rotational property directly and therefore the security of the SM3 compression function, at this stage of analysis, does not seem to be directly affected.

Two 32-bit words $X, Y$ are said to be rotational if $X=Y \lll n$. Let messages $W$ and $W^{*}$ satisfy $W_{1}^{*}=W_{0} \lll 1, W_{2}^{*}=W_{1} \lll 1, \ldots, W_{16}^{*}=W_{15} \lll 1$. Below, a procedure for the instant generation of pairs $v, v^{*}$ such that 
Table 7. Example of a quartet for 33 steps of the SM3 compression function

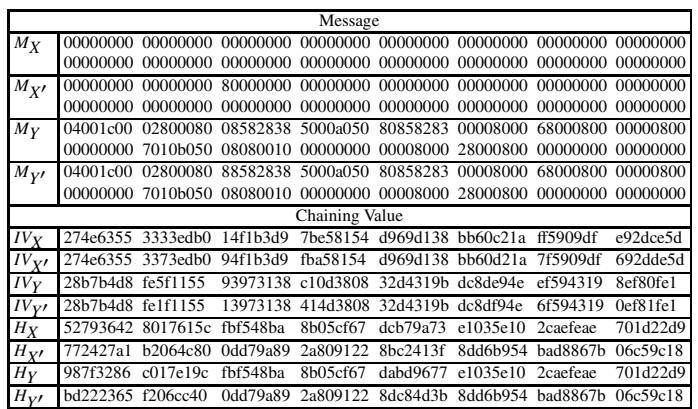

Table 8. An example for a slide-rotational pair for the SM3-XOR compression function

\begin{tabular}{|c|c|}
\hline$A^{1}, B^{1}, \ldots, H^{1}$ & 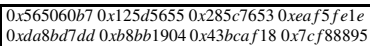 \\
\hline$W_{0}^{1}, \ldots, W_{15}^{1}$ & $\begin{array}{l}0 x 8 f 450 b b d \quad 0 x 4 a 0 c 99220 x 73 d d 44 f 80 x 9 e c e a a f 8 \\
0 x 33 b 13 e 200 x b 59 d 9 c 330 x 6 b 5 a 5 f 230 x c 0 d 2 b 468 \\
0 x 7 a 9 a 1 e 160 x a f f 628780 x 3 \text { fbb } 801 f 40 x 75278787 \\
0 x a c 0 b 849 e \quad 0 x 498 f 30450 x 62687 c 150 x d 3498 e b\end{array}$ \\
\hline$A^{2}, B^{2}, \ldots, H^{2}$ & $\begin{array}{l}0 \times 24 \text { baacaa } 0 \times 53285 c 760 x d 5 \text { ebfc } 3 d \text { oxdf } 1 e e 2 a 6 \\
0 x 717632090 x 2 b c 610 \text { ef } 0 x f 9 f 1112 a \text { 0xffeb } 86 a 4\end{array}$ \\
\hline$W_{0}^{2}, \ldots, w_{15}^{2}$ & $\begin{array}{l}0 x 7 e f a 75420 x 1 e 8 a 177 b 0 x 941932440 x e 7 b a 89 f 0 \\
0 x 3 d 9 d 55 f 10 x 67627 c 400 x 6 b 3 b 38670 x d 6 b 4 b e 46 \\
0 x 81 a 568 d 10 x f 5343 c 2 c 0 x 5 f e c 50 f 10 x 7 f 7603 e 8 \\
\text { 0xea } 4 f 0 f 0 e \quad 0 x 5817093 d 0 x 931 e 608 a 0 x c 4 d 0 f 82 a\end{array}$ \\
\hline
\end{tabular}

$$
\begin{gathered}
v_{1}^{*}=v_{0} \lll 1, v_{2}^{*}=v_{1} \lll 8, v_{3}^{*}=v_{2} \lll 1 \\
v_{5}^{*}=v_{4} \lll 1, v_{6}^{*}=v_{5} \lll 18, v_{7}^{*}=v_{6} \lll 1 \\
V_{1}^{*}=V_{0} \lll 1, V_{2}^{*}=V_{1} \lll 8, V_{3}^{*}=V_{2} \lll 1 \\
V_{5}^{*}=V_{4} \lll 1, V_{6}^{*}=V_{5} \lll 18, V_{7}^{*}=V_{6} \lll 1
\end{gathered}
$$

is provided, where $V=\operatorname{SM} 3-\operatorname{XOR}(v, W), V^{*}=\operatorname{SM} 3-\operatorname{XOR}\left(v^{*}, W^{*}\right)$ and $v_{i}, V_{i}$ for $0 \leq$ $i \leq 7$ denote $i$-th 32-bit word in the $v$ and $V$, respectively. For a random function, a random $(v, W),\left(v^{*}, W^{*}\right)$ satisfying the above constraints will yield the corresponding $V$ and $V^{*}$ with probability $2^{-6 \times 32}=2^{-192}$, since (4) imposes 632 -bit conditions on $V, V^{*}$.

\subsection{Constructing a Slide-Rotational Pair}

In this section, step $i$ denotes the transformation from $\left(A_{i}, B_{i}, C_{i}, D_{i}, E_{i}, F_{i}, G_{i}, H_{i}\right)$ to $\left(A_{i+1}, B_{i+1}, C_{i+1}, D_{i+1}, E_{i+1}, F_{i+1}, G_{i+1}, H_{i+1}\right)$. For example by step 0 the first compression function step is denoted. We start by the following observations:

- The slide rotational messages expand to slide-rotational expanded messages with probability 1 . In particular, fix $W_{0}, \ldots, W_{15}$ and let

$$
W_{1}^{*}=W_{0} \lll 1, W_{2}^{*}=W_{1} \lll 1, \ldots, W_{16}^{*}=W_{15} \lll 1
$$

After expanding both $W$ and $W^{*}$, we have $W_{i+1}^{*}=W_{i} \lll 1$, for $i=\{0,1, \ldots, 62\}$ and also $W_{i+1}^{*}=W_{i}^{\prime} \lll 1$, for $i=\{0,1, \ldots, 66\}$. 
- We recall that $T_{i}, 0 \leq i \leq 63$ are the step constants. If we have

$$
\begin{aligned}
& W_{i+1}^{*}=W_{i} \lll 1, W_{i+1}^{* *}=W_{i}^{\prime} \lll 1, T_{i+1}=T_{i} \lll 1 \\
& A_{i+1}^{*}=A_{i} \lll 1, B_{i+1}^{*}=B_{i} \lll 1, \ldots, H_{i+1}^{*}=H_{i} \lll 1
\end{aligned}
$$

for $i=k$, then (7) will also hold for $i=k+1$, where $k=0, \ldots, 62$.

The observations above suggest that sliding can be introduced, as depicted in Fig. 2

Namely, consider randomly initializing $W$ and letting $W^{*}$ satisfy (5). Moreover, $A_{0}, B_{0} \ldots, H_{0}$ is chosen randomly and the inner state registers after the first step in the second instance of the hash function are initialized according to (7). Then, until step 15 , due to (6), the rotational property in the inner state registers will be preserved. Once the two instances reach steps 15 and 16, respectively, a different step transformation is applied in the two instances and the rotational property may discontinue. This problem is bypassed by starting from the middle, i.e., by populating the inner states entering the critical steps 15 and 16 (see Fig.2).

\subsection{Bypassing Steps 15 and 16}

The idea is to start by populating the inner states entering the critical steps 15 and 16 (see Fig. 2). In particular, a rotational pair $\left(A_{15}, \ldots, H_{15}\right),\left(A_{16}^{*}, \ldots, H_{16}^{*}\right)$ is carefully chosen so that $\left(A_{16}, \ldots, H_{16}\right)$ and $\left(A_{17}^{*}, \ldots, H_{17}^{*}\right)$ satisfy relation (7). It should be noted that the rotational property may be destroyed only between $A_{16}$ and $A_{17}^{*}$ and between $E_{16}$ and $E_{17}^{*}$, since the other registers go through identical rotational-preserving transformations in step 15 and step 16. As for $A_{16}$ and $A_{17}^{*}$, for the purpose of tracking the possible rotational disturbance between the two registers, the equation to compute these two registers can be rewritten as

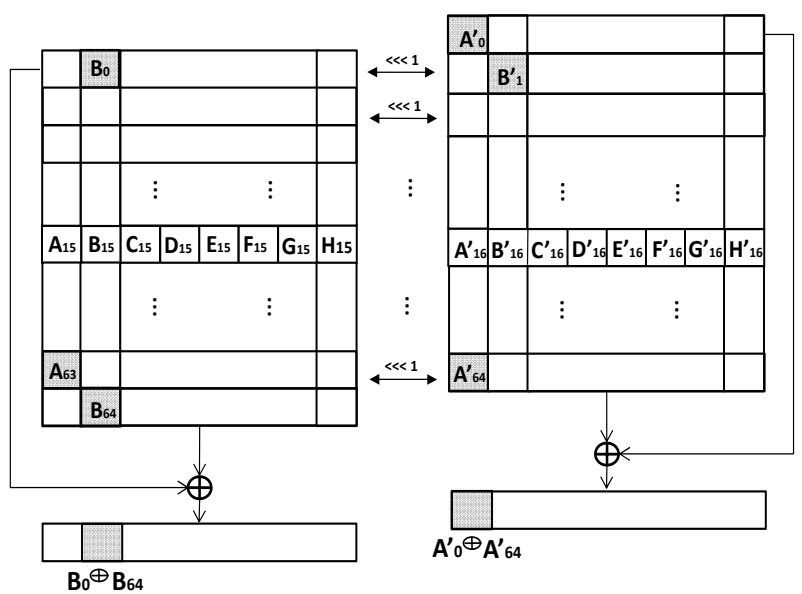

Fig. 2. The slide-rotational attack against SM3-XOR 


$$
\begin{aligned}
& A_{16}=F F_{15}\left(A_{15}, B_{15}, C_{15}\right) \oplus\left(T_{15} \lll 22\right) \oplus \alpha \\
& A_{17}^{*}=F F_{16}\left(A_{16}^{*}, B_{16}^{*}, C_{16}^{*}\right) \oplus\left(T_{16} \lll 23\right) \oplus \alpha^{*}
\end{aligned}
$$

where $\alpha=D_{15} \oplus W_{15} \oplus W_{19} \oplus\left(\left(\left(A_{15} \lll 12\right) \oplus E_{15}\right) \lll 7\right) \oplus\left(A_{15} \lll 12\right)$ and $\alpha^{*}=$ $D_{16}^{*} \oplus W_{16}^{*} \oplus W_{20}^{*} \oplus\left(\left(\left(A_{16}^{*} \lll 12\right) \oplus E_{16}^{*}\right) \lll 7\right) \oplus\left(A_{16}^{*} \lll 12\right)$. Since (7) and (6) hold for $i=15, \alpha^{*}=\alpha \lll 1$. Therefore, to have $A_{16}$ and $A_{17}^{*}$ be a rotational pair, it suffices to make $F F_{15}\left(A_{15}, B_{15}, C_{15}\right) \oplus\left(T_{15} \lll 22\right)$ and $F F_{16}\left(A_{16}^{*}, B_{16}^{*}, C_{16}^{*}\right) \oplus\left(T_{16} \lll 23\right)$ satisfy the rotational property. After expressing $A_{16}^{*}, B_{16}^{*}, C_{16}^{*}$ in terms of $A_{15}, B_{15}, C_{15}$ and using that $F F_{15}$ and $F F_{16}$ preserve the rotational property, the condition can be expressed in terms of $A_{15}, B_{15}, C_{15}$ as follows:

$$
F F_{15}\left(A_{15}, B_{15}, C_{15}\right) \oplus F F_{16}\left(A_{15}, B_{15}, C_{15}\right)=\left(T_{15} \oplus T_{16}\right) \lll 22
$$

When applied on 1-bit values $X, Y$ and $Z$, the equation $F F_{15}(X, Y, Z) \oplus F F_{16}(X, Y, Z)=0$ is satisfied for 2 out of $8(X, Y, Z)$ values. Since the Hamming weight of the right-hand side of $(10)$ is equal to 14 , the number of solutions to the equation is $2^{18} \times 6^{14}=$ $2^{32} \times 3^{14}$. As for preserving the rotational property between $E_{16}$ and $E_{17}^{*}$, developing the registers as in (8) and then forming the equation of the form (10) yields that the number of solutions $E_{15}, F_{15}$ and $G_{15}$ is $4^{32}=2^{64}$. Therefore, the number of solutions for $\left(A_{15}, \ldots, H_{15}\right)$ that pass the disturbance in steps 15 and 16 is $2^{32} \times 3^{14} \times 2^{64} \times 2^{64} \approx 2^{182.19}$, since $D_{15}$ and $H_{15}$ are free variables. For such pairs, it follows that relations (4) are satisfied.

When instead of SM3-XOR, the SM3 compression function is considered, this property turns into a probabilistic one. Following [15], if $p_{r}=P[(x \lll r)+(y \lll r)=$ $(x+y) \lll r]$ where $x$ and $y$ are 32-bit words, then $p_{1}=2^{-1.415}$. Since there exists 8 additions in one SM3 step, the probability that one step and its corresponding slided step will preserve the rotational property is given by $\left(p_{1}\right)^{8}=2^{-11.320}[15]$.

\section{Conclusions}

In this paper, we have shown an application of the boomerang-style attack on the stepreduced SM3 compression function. In particular, we presented distinguishing attacks for 32 steps of the compression function with complexity $2^{14.4}, 33$ steps with complexity $2^{32.4}, 34$ steps with complexity $2^{53.1}$ and 35 steps with complexity $2^{117.1}$. Our results suggest that 35-step SM3 compression does not behave randomly. In the second part of the paper, a slide-rotational property of SM3-XOR function is exposed and an example of a slide-rotational pair for SM3-XOR compression function is given.

Acknowledgment. The authors would like to thank the anonymous reviewers for valuable comments that helped improve the presentation of the paper. The work of Gaoli Wang and Yanzhao Shen is supported by the National Natural Science Foundation of China (No. 61103238), Chen Guang project (supported by Shanghai Municipal Education Commission and Shanghai Education Development Foundation), the Fundamental Research Funds for the Central Universities. The work of Aleksandar Kircanski and Amr Youssef is supported by the Natural Sciences and Engineering Research Council of Canada (NSERC). 


\section{References}

1. Specification of SM3 cryptographic hash function (in Chinese), http://www.oscca.gov.cn/UpFile/20101222141857786.pdf/

2. Zou, J., Wu, W., Wu, S., Su, B., Dong, L.: Preimage Attacks on Step-Reduced SM3 Hash Function. In: Kim, H. (ed.) ICISC 2011. LNCS, vol. 7259, pp. 375-390. Springer, Heidelberg (2012)

3. Aumasson, J.P.: Zero-sum Distinguishers. Rump session talk at CHES 2009 (2009), http://131002.net/data/papers/AM09.pdf

4. Wagner, D.: The Boomerang Attack. In: Knudsen, L.R. (ed.) FSE 1999. LNCS, vol. 1636, pp. 156-170. Springer, Heidelberg (1999)

5. Biryukov, A., Nikolić, I., Roy, A.: Boomerang Attacks on BLAKE-32. In: Joux, A. (ed.) FSE 2011. LNCS, vol. 6733, pp. 218-237. Springer, Heidelberg (2011)

6. Lamberger, M., Mendel, F.: Higher-Order Differential Attack on Reduced SHA-256. Cryptology ePrint Archive: Report 2011/037, http://eprint.iacr.org/

7. Biryukov, A., Lamberger, M., Mendel, F., Nikolić, I.: Second-Order Differential Collisions for Reduced SHA-256. In: Lee, D.H. (ed.) ASIACRYPT 2011. LNCS, vol. 7073, pp. 270287. Springer, Heidelberg (2011)

8. Mendel, F., Nad, T.: Boomerang Distinguisher for the SIMD-512 Compression Function. In: Bernstein, D.J., Chatterjee, S. (eds.) INDOCRYPT 2011. LNCS, vol. 7107, pp. 255-269. Springer, Heidelberg (2011)

9. Sasaki, Y.: Boomerang Distinguishers on MD4-Family: First Practical Results on Full 5-Pass HAVAL. In: Miri, A., Vaudenay, S. (eds.) SAC 2011. LNCS, vol. 7118, pp. 1-18. Springer, Heidelberg (2012)

10. Sasaki, Y., Wang, L.: 2-Dimension Sums: Distinguishers Beyond Three Rounds of RIPEMD128 and RIPEMD-160, http://eprint.iacr.org/2012/049.pdf

11. Aumasson, J.-P., Çalık, Ç., Meier, W., Özen, O., Phan, R.C.-W., Varıc1, K.: Improved Cryptanalysis of Skein. In: Matsui, M. (ed.) ASIACRYPT 2009. LNCS, vol. 5912, pp. 542-559. Springer, Heidelberg (2009)

12. Leurent, G., Roy, A.: Boomerang Attacks on Hash Function Using Auxiliary Differentials. In: Dunkelman, O. (ed.) CT-RSA 2012. LNCS, vol. 7178, pp. 215-230. Springer, Heidelberg (2012)

13. Chen, J., Jia, K.: Improved Related-Key Boomerang Attacks on Round-Reduced Threefish512. In: Kwak, J., Deng, R.H., Won, Y., Wang, G. (eds.) ISPEC 2010. LNCS, vol. 6047, pp. 1-18. Springer, Heidelberg (2010)

14. Yu, H., Chen, J., Wang, X.: The Boomerang Attacks on the Round-Reduced Skein-512. In: Knudsen, L.R., Wu, H. (eds.) SAC 2012. LNCS, vol. 7707, pp. 288-304. Springer, Heidelberg (2012)

15. Khovratovich, D., Nikolić, I.: Rotational Cryptanalysis of ARX. In: Hong, S., Iwata, T. (eds.) FSE 2010. LNCS, vol. 6147, pp. 333-346. Springer, Heidelberg (2010)

16. Biryukov, A., Wagner, D.: Slide Attacks. In: Knudsen, L.R. (ed.) FSE 1999. LNCS, vol. 1636, pp. 245-259. Springer, Heidelberg (1999)

17. Yoshida, H., Biryukov, A.: Analysis of a SHA-256 Variant. In: Preneel, B., Tavares, S. (eds.) SAC 2005. LNCS, vol. 3897, pp. 245-260. Springer, Heidelberg (2006)

18. Wang, X., Yu, H.: How to Break MD5 and Other Hash Functions. In: Cramer, R. (ed.) EUROCRYPT 2005. LNCS, vol. 3494, pp. 19-35. Springer, Heidelberg (2005)

19. Wagner, D.: A Generalized Birthday Problem. In: Yung, M. (ed.) CRYPTO 2002. LNCS, vol. 2442, pp. 288-303. Springer, Heidelberg (2002) 